\title{
Efecto de la concentración de silicato de sodio y ácido fosfórico en la reducción de la temperatura de cocción de las baldosas cerámicas
}

No. 2

\section{Effect of the concentration of sodium silicate and phosphoric acid in reducing the firing temperature of the ceramic tiles}

Forma de citar: R. Monroy, B. Ramón, Y. A. Romero, "Efecto de la concentración de silicato de sodio y ácido fosfórico en la reducción de la temperatura de cocción de las baldosas cerámicas”, Respuestas, vol. 19, no. 2, pp. 25-31, 2014.

Recibido:

Marzo 4 de 2014

Aceptado:

Mayo 8 de 2014

Ingeniero de Producción Industrial richard.monroy@unilibrecucuta. edu.co

Universidad Libre Seccional Cúcuta-Colombia

${ }^{2}$ Doctor en Ingeniería de Materiales

Universidad de Pamplona Pamplona- Colombia

${ }^{3}$ Especialista en Gestión de Proyectos

Universidad Libre Seccional Cúcuta-Colombia

\author{
Richard Monroy-Sepúlveda ${ }^{1}$, Bladimir Ramón- Valencia ${ }^{2}$, Yebrail Alexis Romero- Arcos ${ }^{3}$
}

\section{Resumen}

Objetivo: determinar la reducción de la temperatura de cocción en baldosas cerámicas en función de la concentración de silicato de sodio y ácido fosfórico presente en la formulación de mezclas de pasta arcillosa. Métodos: la investigación se llevó a cabo utilizando una muestra de arcilla de la formación geológica Guayabo del Área Metropolitana de Cúcuta. Para la elaboración de las probetas se adicionaron a la arcilla tres concentraciones de ácido fosfórico a 0,5\%, 1,0\% y 1,5\% en peso, conjuntamente con tres concentraciones de silicato sódico hidratado de $1,0 \%, 2,0 \%$ y $3,0 \%$ en peso respectivamente. Las mezclas se molturaron por vía seca y se realizó el amasado manual y conformado en extrusora. Las probetas obtenidas se secaron en una estufa de secado a $110 \stackrel{\circ}{ } \mathrm{C}$ y sinterizaron en un horno eléctrico a cinco temperaturas máximas $\left(650^{\circ} \mathrm{C}, 680^{\circ} \mathrm{C}, 700^{\circ} \mathrm{C}, 750^{\circ} \mathrm{C}\right.$ y $\left.880^{\circ} \mathrm{C}\right)$. Posteriormente se aplicaron ensayos de laboratorio para determinar la absorción de agua, contracción en cocido y resistencia a la flexión de las probetas. Resultados: los resultados permitieron afirmar que la adición de estos dos aditivos aceleraron la gresificación de la arcilla utilizada, reduciendo los ciclos y temperatura de sinterización de las piezas cerámicas conformadas, lo que hace inferir que es una alternativa en la reducción del consumo de energía térmica empleada en el proceso de cocción de las empresas fabricantes de baldosas cerámicas. Conclusión: repercutiendo en la disminución del impacto ambiental generado por emisiones de $\mathrm{CO}_{2} \mathrm{y}$ material particulado. ISSN 0122-820X

Palabras clave: Ácido Fosfórico, Arcilla, Baldosas, Sinterización, Silicato de Sodio.

\section{Abstract}

Objective: Determining the reduction in the firing temperature 
No. 2

Julio-Diciembre 2014 ISSN 0122-820X

PP: 25-31

ceramic tiles according to the concentration of sodium silicate and phosphoric acid present in the formulation of mixtures of clay paste. Methods: the research was conducted using a sample of clay Guayabo geological formation of the Metropolitan Area of Cucuta. For the preparation of the samples were added to the clay three concentrations of phosphoric acid at $0.5 \%, 1,0 \%$ and $1,5 \%$ by weight , together with three concentrations of hydrated sodium silicate 1,0\%, 2,0\% and 3,0 $\%$ by weight respetivamente. The mixtures were ground dry processing and manual kneading and shaping was performed using a laboratory extruder biscuit. The produced samples were dried in a drying oven at $110{ }^{\circ} \mathrm{C}$ and sintered in an oven five maximum temperatures $\left(650{ }^{\circ} \mathrm{C}\right.$, $680^{\circ} \mathrm{C}, 700^{\circ} \mathrm{C}, 750^{\circ} \mathrm{C}$ and $\left.880^{\circ} \mathrm{C}\right)$. Subsequently laboratory tests were performed to determine the water absorption and cooked contraction in flexural strength of the test pieces. Results: the results allowed to state that the addition of these two additives accelerated the vitrification of the clay used, reducing the cycles and sintering temperature of the ceramic molded parts, making infer that it is an alternative in reducing the consumption of thermal energy used in the cooking process of the manufacturers of ceramic tiles. Conclusion: which influence the reduction of the environmental impact caused by emissions of $\mathrm{CO}_{2}$ and particulate matter.

Keywords: Phosphoric Acid, Clay, Tiles, Sodium Silicate, Sintering.

\section{Introducción}

\section{Elárea metropolitana de Cúcuta se caracteriza} por la gran concentración de empresas dedicadas a la extracción y transformación de la arcilla en productos cerámicos de construcción; la región posee un gran potencial de materias primas arcillosas concentradas mayoritariamente en la formación geológica Guayabo [1].
La industria cerámica regional se caracteriza por el uso de hornos de llama invertida para la sinterización de las diferentes piezas de arcilla elaboradas, siendo esta tecnología la de mayor frecuencia de trabajo en la región, teniendo en cuenta que en este proceso se emplea como combustible carbón mineral el cual es un combustible fósil que tiene como consecuencia la emisión de dióxido de carbono $\left(\mathrm{CO}_{2}\right)$, que es uno de los gases de efecto invernadero responsables del cambio climático y del calentamiento global del planeta [2].
Aunque los aspectos económicos son de gran influencia a la hora de elegir el sistema productivo y tecnológico a emplear [3], la protección del medio ambiente actualmente juega un papel muy importante en el éxito de las empresas fabricantes de esta tipología de productos. Los empresarios de la región dedicados a esta industria han conformado sus empresas sin tener claridad de los impactos ambientales generados por este tipo reglón de la economía, y como realizar gestión ambiental para reducir los efectos nocivos.

La presente investigación se ha realizado como un aporte a esta problemática tanto para los empresarios como para las unidades académicas e investigativas que guardan relación con el tema, con el fin de plantear nuevas alternativas para el uso racional de combustibles fósiles y la reducción térmica en los procesos de sinterización de la industria fabricantes de baldosas cerámicas de la región y a nivel nacional, a partir del conocimiento y dominio de la ciencia e ingeniería de 
materiales, que permitan establecer una nueva pasta cerámica (formulación de pasta cerámica acuosa con aditivos) que le confieran propiedades de gresificación a bajas temperaturas con menor ciclo de cocción [4], sin perder las características de un producto con baja absorción de agua y alta resistencia mecánica.

\section{Materiales y métodos}

La investigación realizada es de tipo experimental, la cual se llevó a cabo en las instalaciones de la empresa Cerytec Ltda. ubicada en el Municipio de Villa del Rosario Norte de Santander.

\subsection{Materiales}

La Arcilla tomada como materia prima se extrajo del área metropolitana de San José de Cúcuta, Norte de Santander correspondiente al grupo Guayabo de la Mina Támesis, ubicada en jurisdicción del municipio de Los Patios; utilizado un muestreo de tipo intencionado.

El análisis químico y mineralógico, fue realizado por el Grupo de Investigaciones en Minerales, Biohidrometalurgia y Ambiente de la Universidad Industrial de Santander utilizando la técnica de Difracción de Rayos X (DRX) [5], los cuales se presentan en la tabla I. A su vez se pueden observar en la figura 1, los análisis mineralógicos de las arcilla Támesis.

Tabla I. Análisis químico de la muestra arcillosa Grupo Guayabo de la Mina Támesis

\begin{tabular}{|c|c|c|c|}
\hline \multicolumn{4}{|c|}{ COMPOSICIÓN ELEMENTAL } \\
\hline \multirow{2}{*}{$\begin{array}{l}\text { ELEMENTO } \\
\mathrm{Si02}\end{array}$} & \multicolumn{2}{|c|}{ RESULTADO } & \multirow{2}{*}{$\begin{array}{l}\text { DESVIACIÓN ESTÁNDAR } \\
(0.146)\end{array}$} \\
\hline & 60.950 & $\div$ & \\
\hline A1203 & 21.434 & $\div$ & \\
\hline $\mathrm{Fe} 203$ & 5.310 & $\div$ & $(0.011)$ \\
\hline $\mathrm{K} 20$ & 1.956 & * & $(0.013)$ \\
\hline s03 & 1.504 & ; & $(0.020)$ \\
\hline $\mathrm{Na} 20$ & 1.271 & $\%$ & $(0.740)$ \\
\hline $\mathrm{Ti} 02$ & 0.973 & $\div$ & $(0.010)$ \\
\hline Mg0 & 0.396 & $\%$ & $(0.102)$ \\
\hline $\mathrm{CaO}$ & 0.196 & $\div$ & $(0.006)$ \\
\hline Mno & 0.035 & $\div$ & $(0.002)$ \\
\hline v205 & 0.033 & * & $(0.004)$ \\
\hline $2 \mathrm{r} 02$ & 0.023 & * & $(0.000)$ \\
\hline $2 \mathrm{no}$ & 0.018 & ; & $(0.001)$ \\
\hline P205 & 0.009 & ; & $(0.024)$ \\
\hline $\mathrm{Rb} 20$ & 0.009 & $\div$ & $(0.000)$ \\
\hline Sro & 0.009 & ; & $(0.000)$ \\
\hline Y203 & 0.003 & $:$ & $(0.000)$ \\
\hline $\mathrm{CO2}$ & 5.870 & ; & $(-\cdots)$ \\
\hline
\end{tabular}

Fuente: Grupo de Investigaciones en Minerales, biohidrometalurgia y Ambiente de la Universidad Industrial de Santander.

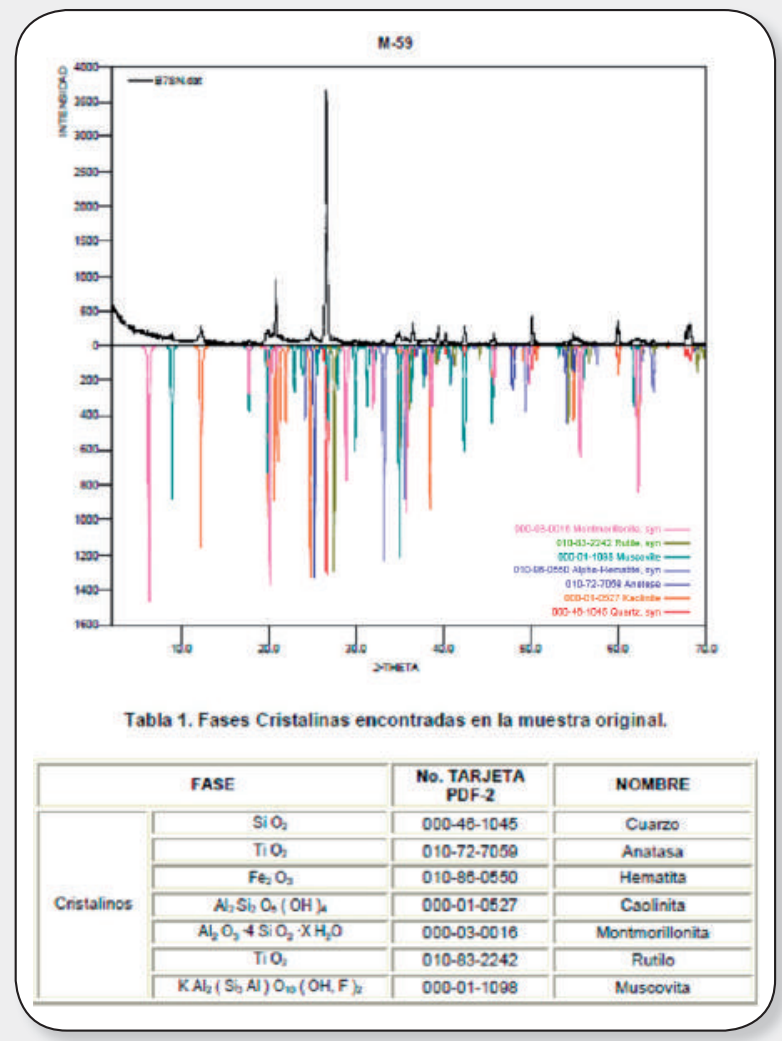

Julio-Diciembre 2014

ISSN 0122-820X

PP: $25-31$

Figura 1. Análisis minerológico de la muestra arcillosa Grupo Guayabo de la Mina Támesis.

Fuente: Grupo de Investigaciones en Minerales, biohidrometalurgia y Ambiente de la Universidad Industrial de Santander.

Adicionalmente se empleó ácido fosfórico grado analítico, solución acuosa al $85 \%$ y al $75 \%$ marca Merck; conjuntamente silicato sódico hidratado grado analítico marca OPPAC S.A.

\subsection{Métodos}

Inicialmente la arcilla o materia prima fue sometida al proceso de reducción de tamaño de partícula, se molturaron por vía seca en el molino de discos. Al material molido por vía seca se le realizó un proceso de tamizado utilizando el tamiz malla 12 serie Tyler de uso común en la industria cerámica regional que utiliza este sistema de molienda. Posteriormente la muestra de arcilla se secó en la estufa de secado eléctrica marca Cerytec con capacidad de $200{ }^{\circ} \mathrm{C}$, hasta alcanzar peso constante. Se determinó el índice de plasticidad por límites de consistencia de Atteberg. Esta técnica consiste en determinar la plasticidad de una arcilla con diferentes contenidos de agua [6], cuando se la golpea varias veces la masa plástica en capsula 
No. 2

Julio-Diciembre 2014 ISSN 0122-820X

PP: 25-31
Casagrande. Los resultados del índice de plasticidad la arcilla Támesis se presenta en la tabla II, esta prueba se realizó en la Universidad Francisco de Paula Santander, Laboratorio de Suelos [7].

Tabla II. Índices de plasticidad de la muestra arcillosa utilizada

\begin{tabular}{|c|c|}
\hline Tipo de muestra & Índice de plasticidad \% \\
\hline $\begin{array}{c}\text { Arcilla roja Támesis, Grupo o formación } \\
\text { Guayabo. }\end{array}$ & 27,0 \\
\hline
\end{tabular}

Fuente: Laboratorio de Suelos, Universidad Francisco de Paula Santander.

De acuerdo con el resultado obtenidodel índice de plasticidad, se humectó manualmente la arcilla estudiada conjuntamente con los aditivos y se mantuvieron durante 2 horas para conseguir una adecuada homogeneización. La fabricación de las probetas fue por el método de conformado de extrusión, para éste proceso se usó la extrusora manual de laboratorio sin vacío modelo Taller Cerámico con capacidad de $1 \mathrm{Kg}$, donde la humedad de la mezcla antes de entrar a la extrusora fue de $26,0 \%$ y de $18,0 \%$ a la salida de ésta. Se prepararon 6 probetas por duplicidad (Ver figura 2), donde el porcentaje en peso de ácido fosforico estuvo en los rangos de: $0,5 \%, 1,0 \%$ y $1,5 \%$, en la mezclas uno, dos y tres, conjuntamente con el silicato sódico hidratado que fue de: 1,0\%, 2,0\% y 3,0\% respectivamente. (Ver Tabla III).

Tabla III. Porcentaje en peso de ácido fosfórico y silicato de sodio en mezclas de las probetas

\begin{tabular}{|c|c|c|c|}
\hline Mezcla & Arcilla \% & $\begin{array}{c}\text { Ácido fosfórico } \\
\%\end{array}$ & Silicato de sodio \% \\
\hline M1 & 100 & 0,0 & 0,0 \\
\hline M2 & 98,00 & 0,5 & 1,5 \\
\hline M3 & 97 & 1,0 & 2,0 \\
\hline M4 & 95,5 & 1,5 & 3,0 \\
\hline
\end{tabular}

Fuente: Autores
Las probetas obtenidas se secaron en estufa a una temperatura de $110^{\circ} \mathrm{C}$ con una precisión de $+1-5^{\circ} \mathrm{C}$ durante 4 horas y posteriormente; se sinterizarón en un horno túnel eléctrico marca Cerytec y modelo CYT-KLIN a cinco temperaturas máximas $\left(650{ }^{\circ} \mathrm{C}, 680{ }^{\circ} \mathrm{C}\right.$, $700^{\circ} \mathrm{C}, 750^{\circ} \mathrm{C}$ y $\left.880^{\circ} \mathrm{C}\right)$ [4].

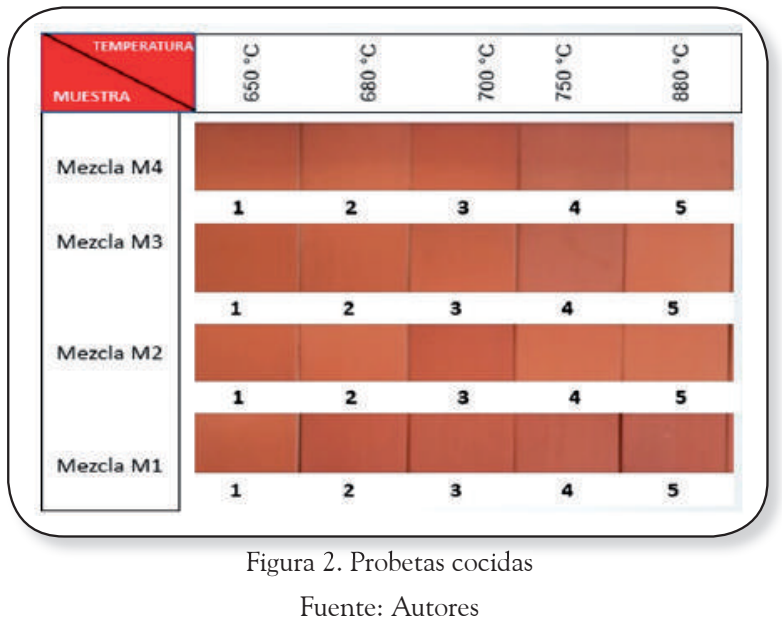

Después del ciclo de cocción, en cada lote las probetas se pesaron y midieron, una vez alcanzaron la temperatura ambiente. Finalmente se realizó la prueba de contracción en cocido basado en la norma internacional ASTM [8]; absorción de agua por ebullición, y resistencia a la flexión utilizando la metodología establecida en las normas técnicas colombianas NTC 4321-3 [9] y, NTC 4321-4 [10].

\section{Resultados y análisis}

En la Tabla V, se presentan los datos promedios de las contracciones en cocido, porcentaje de absorción de agua y resistencia a la flexión promedio de las diferentes mezclas. De éstos, se aprecia que tanto la contracción en cocido como la resistencia a la flexión aumentan al agregar más concentración de ácido fosfórico y silicato de sodio, mientras que la absorción de agua disminuye. 
Richard Monroy-Sepúlveda, Bladimir Ramón- Valencia, Yebrail Alexis Romero- Arcos

Tabla IV. Datos promedios de ensayos a las mezclas seleccionadas

\begin{tabular}{|c|c|c|c|c|}
\hline Mezclas & $\begin{array}{c}\text { Temperatura } \\
\left({ }^{\circ} \mathrm{C}\right)\end{array}$ & $\begin{array}{c}\text { Contracción en cocido } \\
(\%)\end{array}$ & $\begin{array}{c}\text { Absorción de Agua } \\
(\%)\end{array}$ & $\begin{array}{c}\text { Resistencia a la flexión } \\
\left(\mathrm{N} / \mathrm{mm}^{2}\right)\end{array}$ \\
\hline \multirow{5}{*}{ M1 } & 650 & 1,10 & 22,30 & 9,0 \\
\hline & 680 & 1,20 & 21,77 & 10,3 \\
\hline & 700 & 1,35 & 20,92 & 10,6 \\
\hline & 750 & 2,00 & 20,18 & 11,9 \\
\hline & 880 & 2,20 & 18,69 & 9,8 \\
\hline \multirow{5}{*}{ M2 } & 650 & 1,34 & 21,13 & 12,0 \\
\hline & 680 & 1,42 & 19,91 & 12,7 \\
\hline & 700 & 1,65 & 19,47 & 13,1 \\
\hline & 750 & 1,73 & 18,89 & 13,7 \\
\hline & 880 & 2,33 & 17,62 & 13,9 \\
\hline \multirow{5}{*}{ M3 } & 650 & 1,35 & 18,94 & 12,4 \\
\hline & 680 & 1,91 & 18,21 & 13,1 \\
\hline & 700 & 2,54 & 17,78 & 13,7 \\
\hline & 750 & 3,23 & 16,09 & 14,1 \\
\hline & 880 & 3,43 & 10,72 & 14,3 \\
\hline \multirow{5}{*}{ M4 } & 650 & 1,38 & 18,74 & 12,5 \\
\hline & 680 & 1,99 & 18,01 & 13,3 \\
\hline & 700 & 2,74 & 17,61 & 13,7 \\
\hline & 750 & 3,43 & 15,00 & 14,2 \\
\hline & 880 & 3,63 & 10,02 & 14,5 \\
\hline
\end{tabular}

Fuente: Autores
Respuestas

Cúcuta-Colombia

Vol. 19

No. 2

Julio-Diciembre 2014 ISSN 0122-820X

PP: $25-31$

En la figura 3 se puede observar la curva de gresificación de las diferentes muestras analizadas, en las cuales se de acuerdo a los datos estudiados se puede afirmar que a medida que se aumenta la adición de ácido fosfórico y silicato de sodio el punto de cocción óptimo de las pasta disminuye; es decir; que la pasta sinteriza a una menor temperatura; teniendo en cuenta que la mezclas M1 y M2 para la temperaturas de cocción evaluadas no sinterizan (ver figura a y b), por lo contrario la muestra M3 y M4 sinterizan a una temperatura promedio de $805^{\circ} \mathrm{C}$ (ver figura c y d).

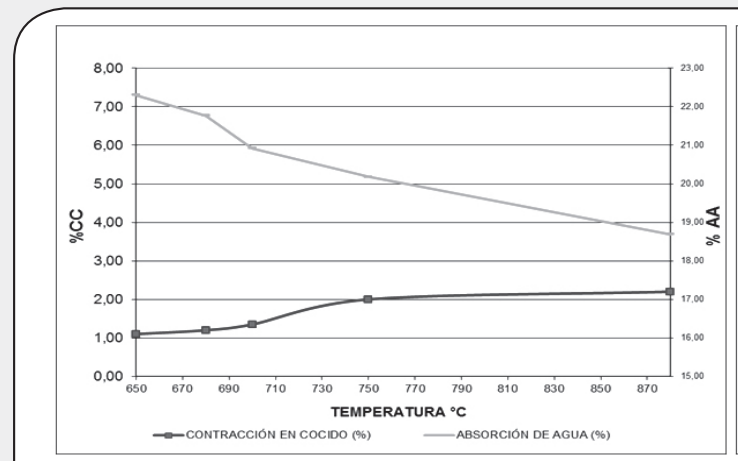

a) Muestra M1

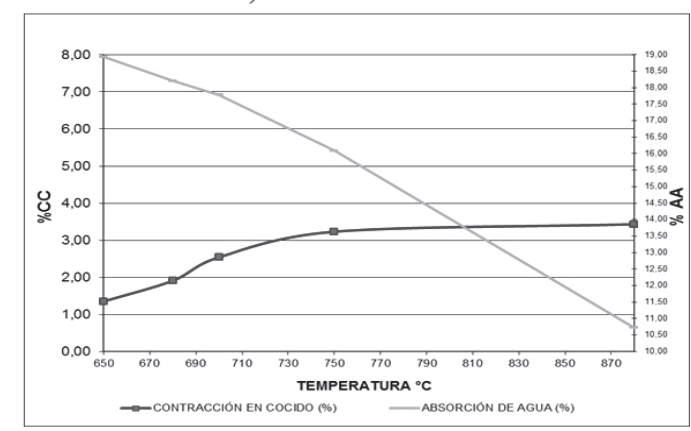

c) Muestra M3

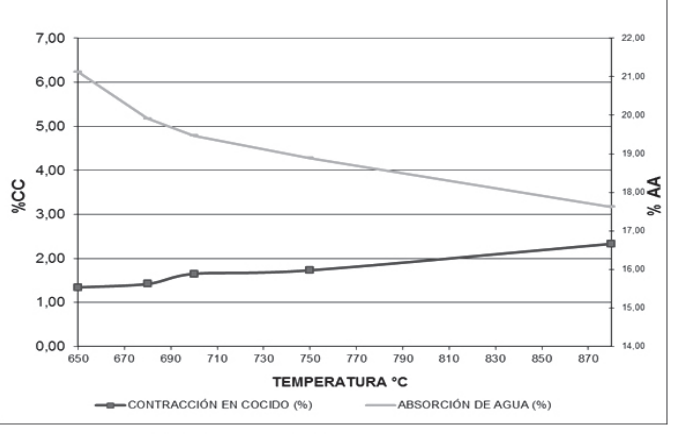

b) Muestra M2

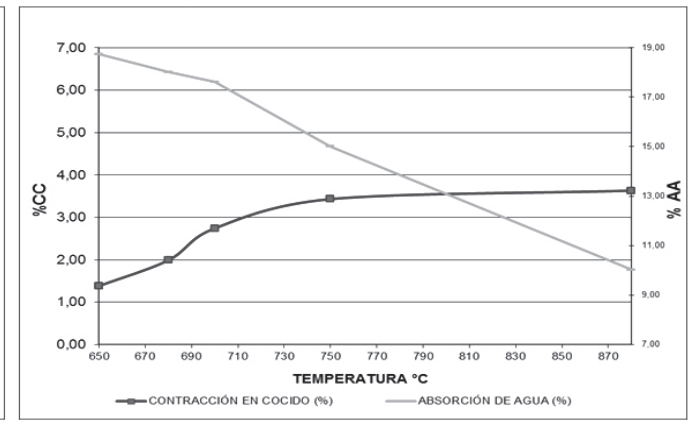

d) Muestra M4

Figura 3. Curva de gresificación de probetas conformadas. 
No. 2

Julio-Diciembre 2014 ISSN 0122-820X

PP: $25-31$
En la figura 4 se presenta los datos reportados de resistencia a la flexión, la cual esta expresada en $\mathrm{N} / \mathrm{mm}^{2}$, evidenciando que la resistencia de la muestra M3 y M4 son similares, lo que permite corroborar que la Sinterización de estas dos pastas es similar, como se había enunciado de antemano en la curva de gresificación, reflejándose en una alta correlación entre esas dos propiedades físicas.

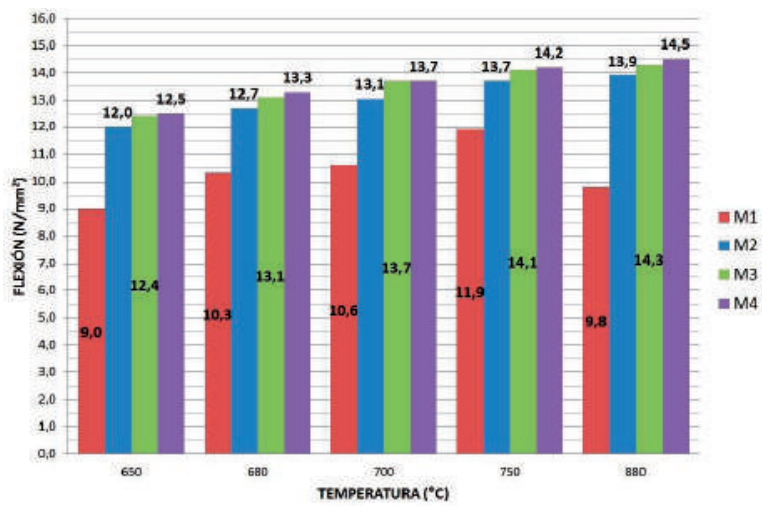

Figura 4. Resistencia a la flexión de probetas conformadas Fuente: Autores

\section{Conclusiones}

se puede afirmar que la investigación permite determinar con certeza una formulación de pasta para extrusión entre arcilla y aditivos (ácido fosfórico y silicato de sodio) para la fabricación de baldosas cerámicas por el método de conformado por extrusión; que plantea un escenario de reducción de los ciclos de sinterización, y que a su vez va permitir el ahorro energético por minimización del consumo de combustible utilizado a través de todo el ciclo de quema, conjuntamente asociado a la reducción de la generación de emisiones, mitigando la contaminación ambiental en la calidad del aire emanado por fuentes fijas.

Se encontró que las muestras M3 (97\% arcilla $1,0 \%$ de ácido fosfórico y 2,0\% silicato de sodio) y M4 (95,5\% arcilla 1,5\% de ácido fosfórico y $3,0 \%$ silicato de sodio), son las recomendables a utilizar para la fabricación de baldosas cerámicas, sabiendo a nivel experimental que dichas muestras tienen un comportamiento similar, y que mejora notablemente el desempeño en la sinterización y sellamiento de poros del producto final, lo que le confiere adecuadas propiedades físico mecánicas, principales características técnicas que deben poseer los revestimientos para pisos y paredes. Esto permite inferir que las baldosas a fabricar bajo el uso de esta formulación garantizarán una disminución aproximada de la temperatura máxima de quema de $200^{\circ} \mathrm{C}$; debido a la gresificación del material cerámico a menores condiciones de temperatura sin deteriorar sus propiedades físicas que caracterizan a estos productos.

\section{Agradecimientos}

Los autores expresan su agradecimiento a la empresa Cerytec Ltda y a la Universidad Libre Seccional Cúcuta por el apoyo brindado para la realización de este trabajo.

\section{Referencias}

[1] Jeskar Ltda, Inventario Geológico Minero, Ambiental, Tecnológico y Empresarial de los Minerales no Energéticos del Norte de Santander. San José de Cúcuta, Colombia, 2000.

[2] E. Monfort, et al. Análisis de consumos energéticos y emisiones de dióxido de carbono en la fabricación de baldosas cerámicas. Boletín de la Sociedad Española de Cerámica y Vidrio. vol. 49, no. 4, pp. 303-310, 2010.

[3] M. Fernández, "Manual sobre fabricación de baldosas, tejas y ladrillos" [DISCO MAGNETICO]. Laboratorio Técnico Cerámico, Igualada, España. 2000.

[4] C. M. Bou, D. Vuji "Method for preparing an aqueous clay paste use thereof in the manufacture of ceramic materials." Patente Número de Publicación Internacional WO 2012/089873 A1, Madrid, España. 2012. 
[5] Universidad Industrial de Santander UIS, Grupo de Investigaciones en Minerales, Biohidrometalurgia y Ambiente, Análisis químico y mineralógicode arcillagrupo Guayabode la Mina Támesis del Área Metropolitana de Cúcuta. Bucaramanga, 2012.

[6] A. M. Querol ViUalba. "Aplicación del método de Pfefferkorn al control de la plasticidad en pastas de extrusión". Boletín de la Sociedad Española de Cerámica y Vidrio, vol. 22, no. 2, pp. 285-325, 1983.

[7] Universidad Francisco de Paula Santander, Laboratorio de Suelos, Análisis de Índice de límites de Atterberg de arcilla grupo Guayabo de la Mina Támesis del Ârea Metropolitana de Cúcuta. 2013.

[8] American Society For Testing And Materials. ASTM C326-03. Standard Test Method for Drying and Firing Shrinkages of Ceramic Whiteware Clays. 2003.

[9] Norma Técnica Colombiana NTC 4321-3 baldosas cerámicas. Parte 3 . Método de ensayo para determinar la absorción de agua, porosidad aparente, densidad relativa aparente y densidad aparente. Bogota D.C. 2005.

[10] Norma Técnica Colombiana NTC 4321-4 baldosas cerámicas. Parte 4. Método de ensayo para determinar 1 resistencia a la flexión y el módulo de rotura. Bogota D.C. 1998. 